Canad. Math. Bull. Vol. 45 (1), 2002 pp. 89-96

\title{
On Gunning's Prime Form in Genus 2
}

\author{
David Grant
}

Abstract. Using a classical generalization of Jacobi's derivative formula, we give an explicit expression for Gunning's prime form in genus 2 in terms of theta functions and their derivatives.

Let $X$ be a compact Riemann surface of genus $g>0$. Let $\tilde{X}$ denote the universal cover of $X, \Pi: \tilde{X} \rightarrow X$ denote the projection, and $\Gamma$ be the group of covering transformations of $\tilde{X}$ over $X$.

By a prime form for $X$ we mean a function on $\tilde{X} \times \tilde{X}$ which is an analytic relatively automorphic function for some prescribed factor of automorphy for the action of $\Gamma$ on each copy of $\tilde{X}$, and which has a simple zero on the diagonal of $\tilde{X} \times \tilde{X}$ and its translates under $\Gamma \times \Gamma$ and has no other zeros. The classic prime form is due to Klein, see $[\mathrm{F}]$ and $[\mathrm{M}]$. In [Gu1] Gunning introduced a different prime form, which has a factor of automorphy that is more closely related to that of theta functions. For applications see [Gu1], [Gu2], [Gu3], [Gu4], [P].

Gunning's prime form is only characterized up to a constant factor by its automorphic and vanishing properties. In [Gu5] Gunning gives an implicit normalization for his prime form (see (2) below) that uses his theory of canonical coordinates on $\tilde{X}$ described in [Gu3].

The purpose of this paper is to give for $g=2$ an explicit expression for Gunning's prime form in terms of genus 2 theta functions and their derivatives. We do so in the Theorem below up to sign: it may well be that the method described below will also suffice to determine the requisite sign, but it seems like a lengthy and perhaps unenlightening exercise to do so. The keys are to use the function theory on the Jacobian of the curve and a generalization of Jacobi's derivative formula due to Rosenhain.

We first recall some basic facts about compact Riemann surfaces and their Jacobians, following the exposition in [Gul]. A marking on $X$ consists of a fixed point $z_{0}$ of $\tilde{X}$, and a canonical basis $\left\{A_{1}, \ldots, A_{g}, B_{1}, \ldots, B_{g}\right\}$ of $H_{1}(X, \mathbb{Z})$. We let $P_{0}=\Pi\left(z_{0}\right)$. With this marking we get an identification between $\Gamma$ and the fundamental group of $X$ based at $P_{0}$, through which we can consider $A_{1}, \ldots, A_{g}, B_{1}, \ldots, B_{g}$ as generators for $\Gamma$.

For any holomorphic differential $\phi$ on $X, \Pi^{*}(\phi)$ is a holomorphic differential on the simply connected space $\tilde{X}$, hence $\Pi^{*}(\phi)=d w$, where $w$ is some analytic function on $\tilde{X}$ which we normalize so that $w\left(z_{0}\right)=0$. Since $\Pi^{*}(\phi)$ is $\Gamma$-invariant, we get a corresponding map $\bar{\phi}: \Gamma \rightarrow \mathbb{C}$ defined by $\bar{\phi}(\gamma)=w(\gamma z)-w(z)$ for any $z \in \tilde{X}$.

Let $\left\{\phi_{1}, \ldots, \phi_{g}\right\}$ be the basis for the space of holomorphic differentials on $X$ normalized so that $\bar{\phi}_{i}\left(A_{j}\right)=\delta_{i j}$. Let $\omega_{i j}=\bar{\phi}_{i}\left(B_{j}\right)$. Then $\Omega=\left[\omega_{i j}\right]_{i, j=1, \ldots, g}$ is the

Received by the editors March 31, 2000.

The author was partially supported by NSF grant DMS- 9303220 .

AMS subject classification: 14K25, 30F10.

(c) Canadian Mathematical Society 2002. 
period matrix of the marked Riemann surface. A standard calculation shows that $\Omega$ is a symmetric $g \times g$ matrix with positive definite imaginary part. Let ${ }^{t} m$ denote the transpose of a matrix $m$, and set $\Phi={ }^{t}\left(\bar{\phi}_{1}, \ldots, \bar{\phi}_{g}\right)$, and $L=\Phi(\Gamma)$. Then $L=\mathbb{Z}^{g}+\Omega \mathbb{Z}^{g}$ is a lattice in $\mathbb{C}^{g}$. The torus $\mathbb{C}^{g} / L$ is the Jacobian $J(X)$ of $X$. Let $\Pi^{*}\left(\phi_{i}\right)=d w_{i}$ with $w_{i}\left(z_{0}\right)=0$. We then define a map $w: \tilde{X} \rightarrow \mathbb{C}^{g}$ by setting $w(z)={ }^{t}\left(w_{1}(z), \ldots, w_{g}(z)\right)$. This induces an embedding $X \rightarrow J(X)$ by setting $w(P)=w(z) \bmod L$, where $z \in \tilde{X}$ is any point such that $\Pi(z)=P$. The image of $X$ under $w$ is denoted $W_{1}$, and for $s<g$ we write $W_{s}$ for the sum of the $s$ terms $W_{1}+\cdots+W_{1}$. We extend $w$ to a map on divisor classes of $X$ by linearity.

For any $v={ }^{t}\left(v_{1}, \ldots, v_{g}\right) \in \mathbb{C}^{g}, a, b \in \frac{1}{2} \mathbb{Z}^{g}$, we define the genus $g$ theta function with characteristic $\left[\begin{array}{l}a \\ b\end{array}\right]$ and period matrix $\Omega$ as

$$
\theta\left[\begin{array}{l}
a \\
b
\end{array}\right](v)=\theta\left[\begin{array}{l}
a \\
b
\end{array}\right](v, \Omega)=\sum_{n \in \mathbb{Z}^{g}} e^{\pi i^{t}(n+a) \Omega(n+a)+2 \pi i^{t}(n+a)(v+b)} .
$$

Note that $\theta\left[\begin{array}{l}a \\ b\end{array}\right](v)$ is analytic in $v$. We let $\theta(v)=\theta\left[\begin{array}{l}0 \\ 0\end{array}\right](v)$. Also $\theta\left[\begin{array}{l}a \\ b\end{array}\right](-v)=$ $e^{4 \pi i^{t} a b} \theta\left[\begin{array}{c}a \\ b\end{array}\right](v)$, so $\theta\left[\begin{array}{l}a \\ b\end{array}\right](v)$ is even or odd depending on whether $e^{4 \pi i^{t} a b}$ is 1 or -1 , and the characteristic $\left[\begin{array}{l}a \\ b\end{array}\right]$ is called even or odd accordingly.

For $\gamma \in \Gamma$, any factor of automorphy $\chi(\ell, v)$ for the action of $L$ on $\mathbb{C}^{g}$ induces the factor of automorphy $\hat{\chi}(\gamma, z)=\chi(\Phi(\gamma), w(z))$ for the action of $\Gamma$ on $\tilde{X}$. For $s \in \mathbb{C}^{g}$, we define the factor of automorphy $\rho_{s}$ for the action of $L$ on $\mathbb{C}^{g}$ by $\rho_{s}\left(\Phi\left(A_{i}\right)\right)=1$, $\rho_{s}\left(\Phi\left(B_{i}\right)\right)=e^{2 \pi i s_{i}}$. Let $\zeta$ be the factor of automorphy for the action of $\Gamma$ on $\tilde{X}$ defined in [Gu2] by $\zeta\left(A_{j}, z\right)=1, \zeta\left(B_{j}, z\right)=e^{-2 \pi i\left(m_{j}+r_{j}+w_{j}(z)\right) / g}$, where $r, m \in \mathbb{C}^{g}$ are defined by $m_{j}=\sum_{k=1}^{g} \omega_{j k}$ and $r_{j}=\sum_{k=1}^{g} \int_{z_{0}}^{A_{k} z_{0}} w_{j}(z) \Pi^{*}\left(\phi_{k}\right)(z)$. Let $\epsilon \in \mathbb{C}^{g}$ be defined by $\epsilon_{i}=\omega_{i i} / 2$.

We can now define Gunning's prime form $q\left(z_{1}, z_{2}\right)$. It is described up to a constant factor as an analytic function on $\tilde{X} \times \tilde{X}$ such that for all $\gamma \in \Gamma$,

$$
q\left(\gamma z_{1}, z_{2}\right)=\hat{\rho}_{w\left(z_{2}\right)}(\gamma) \zeta\left(\gamma, z_{1}\right) q\left(z_{1}, z_{2}\right)
$$

and

$$
q\left(z_{1}, z_{2}\right)=-q\left(z_{2}, z_{1}\right) \text {. }
$$

To normalize $q$, Gunning requires that for any $z, z_{1}, \ldots, z_{g} \in \tilde{X}$,

$$
\begin{array}{r}
\theta\left(r-\epsilon+m+w(z)-w\left(z_{1}\right)-\cdots-w\left(z_{g}\right)\right) \prod_{1 \leq j<k \leq g} q\left(z_{j}, z_{k}\right) \\
=\operatorname{det}\left(w_{j}^{\prime}\left(z_{k}\right)\right)_{1 \leq j, k \leq g} \prod_{1 \leq i \leq g} q\left(z, z_{i}\right),
\end{array}
$$

where the derivatives are taken with respect to the "canonical coordinates" described in [Gu3]; that is

$$
w_{j}^{\prime}\left(z_{k}\right)=\lim _{z_{k}^{\prime} \rightarrow z_{k}} \frac{w_{j}\left(z_{k}\right)-w_{j}\left(z_{k}^{\prime}\right)}{q\left(z_{k}, z_{k}^{\prime}\right)} .
$$


Since the transformation $q \rightarrow \kappa q$ takes $w_{j}^{\prime}\left(z_{k}\right)$ to $w_{j}^{\prime}\left(z_{k}\right) / \kappa$, (2) determines $q$ up to a $\left(\begin{array}{l}g \\ 2\end{array}\right)$-th root of unity.

It follows directly from (1) that for any $\mu \in \mathbb{C}^{g}$, the factor of automorphy of $\theta(v-\mu-\epsilon)$ for the action of $L$ on $\mathbb{C}^{g}$ is

$$
\xi_{\mu}\left(\Phi\left(A_{i}\right), v\right)=1, \xi_{\mu}\left(\Phi\left(B_{i}\right), v\right)=e^{2 \pi i\left(\mu_{i}-v_{i}\right)} .
$$

It follows immediately that

$$
\hat{\xi}_{-r-m}=\zeta^{g}, \quad \xi_{\mu+s}=\rho_{s} \xi_{\mu} .
$$

A fundamental result is Riemann's vanishing theorem, which says that the zeros of $\theta$ modulo $L$ are $-W_{g-1}+r-\epsilon$. Since $\theta$ is an even function, $-W_{g-1}+r-\epsilon=$ $W_{g-1}-r+\epsilon$, so by the Riemann-Roch theorem, $2(r-\epsilon)=k$, where $k$ is the image under $w$ of any canonical divisor of $X$.

Now let $X$ be the Riemann surface defined by the complex points of the genus 2 curve

$$
C: y^{2}=x^{5}+b_{1} x^{4}+b_{2} x^{3}+b_{3} x^{2}+b_{4} x+b_{5}, \quad b_{i} \in \mathbb{C} .
$$

Every genus 2 Riemann surface arises in this way. We first choose an ordering $P_{i}=$ $\left(a_{i}, 0\right), 1 \leq i \leq 5$, for the affine Weierstrass points of $X$. Then we choose a marking for $X$ so that $\Pi\left(z_{0}\right)=P_{0}$ is the point at infinity on the normalization of $C$, and the canonical homology basis is the traditional one employed for hyperelliptic curves with a given ordering of Weierstrass points [M, p. 3.76].

We will be combining the uniformization of $X$ with that of its Jacobian. Most of what we need is given in $[\mathrm{M}]$.

Since $P_{0}$ is a Weierstrass point, $k$ is the origin of $J(X)$, so $r-\epsilon+m=\Omega a+b$, for some $a, b \in \frac{1}{2} \mathbb{Z}^{2}$, and Riemann's vanishing theorem now says that that $\theta\left[\begin{array}{l}a \\ b\end{array}\right](v)$ vanishes for any $v$ in $W_{1}$ modulo $L$. With the traditional choice of canonical basis, $a \equiv\left(\begin{array}{l}1 / 2 \\ 1 / 2\end{array}\right)$ $\bmod 1$, and $b \equiv\left(\begin{array}{l}1 \\ 1 / 2\end{array}\right) \bmod 1[\mathrm{M}, \mathrm{p} .3 .82]$, and $\left[\begin{array}{l}a \\ b\end{array}\right]$ is an odd theta characteristic.

Let $\sigma$ be the matrix such that $\sigma\left(\begin{array}{c}\frac{d x}{y} \\ \frac{x d x}{y}\end{array}\right)=\left(\begin{array}{c}\phi_{1} \\ \phi_{2}\end{array}\right)$. Following [M], we define the differential operators

$$
\left[\begin{array}{c}
D_{2} \\
D_{1}
\end{array}\right]=-{ }^{t} \sigma\left[\begin{array}{c}
\frac{\partial}{\partial v_{1}} \\
\frac{\partial}{\partial v_{2}}
\end{array}\right]
$$

Then if $z \in \tilde{X}-\Pi^{-1}\left(P_{0}\right)$,

$$
D_{x(z)}=D_{2}+x(z) D_{1}
$$

is a differential operator such that if we choose an appropriate local coordinate $z(\rho)$ centered at $z$, then

$$
D_{x(z)} f(v)=\left.\frac{d}{d \rho} f(v+w(z)-w(z(\rho)))\right|_{\rho=0} .
$$

Similarly, if $z \in \Pi^{-1}\left(P_{0}\right)$, then $D_{\infty}=D_{1}$ has the property corresponding to (5). It follows immediately from Riemann's vanishing theorem that for the correct choice of local coordinate $z_{0}(\rho)$ centered at $z_{0}$, that

$$
D_{\infty} \theta(a \Omega+b)=\left.\frac{d}{d \rho} \theta\left(a \Omega+b+w\left(z_{0}\right)-w\left(z_{0}(\rho)\right)\right)\right|_{\rho=0}=0 .
$$


And again, since $\theta(a \Omega+b+w(z))$ vanishes identically, $D_{\infty}(\theta(a \Omega+b+w(z)))$ has the factor of automorphy $\hat{\xi}_{-r-m}=\zeta^{2}$ for the action of $\Gamma$ on $\tilde{X}$. In [Gu1] it is shown that there exists a relatively analytic function $h$ for the factor of automorphy $\zeta$ which vanishes simply at $\Pi^{-1}\left(P_{0}\right)$ and has no other zeros, hence $D_{\infty}(\theta(a \Omega+b+w(z))) / h^{2}$ is a function on $X$ with at most a single, simple pole, so is a constant. Hence $D_{\infty}(\theta(a \Omega+b+w(z)))$ has a double zero at $\Pi^{-1}\left(P_{0}\right)$ and no other zeros, and has a well-defined square root $\psi(z)$. There is an ambiguity of a sign in the definition of $\psi(z)$, but the ambiguity will disappear in the formula (6) below.

We can now calculate Gunning's prime form for $X$ up to constant factor. Let $f\left(z_{1}, z_{2}\right)=\theta\left(w\left(z_{1}\right)-w\left(z_{2}\right)+\Omega a+b\right)$. We then define

$$
\begin{aligned}
Q\left(z_{1}, z_{2}\right) & =\frac{f\left(z_{1}, z_{2}\right) e^{-4 \pi i^{t} a w\left(z_{2}\right)}}{\psi\left(z_{1}\right) \psi\left(z_{2}\right)} \\
& =\frac{\theta\left[\begin{array}{c}
a \\
b
\end{array}\right]\left(w\left(z_{1}\right)-w\left(z_{2}\right)\right)}{\Sigma\left(z_{1}\right) \Sigma\left(z_{2}\right)},
\end{aligned}
$$

where we set $\Sigma(z)=e^{\pi i^{t} a \Omega a / 2+\pi i^{t} a b+2 \pi i^{t} a w(z)} \psi(z)$, so

$$
\Sigma(z)^{2}=e^{2 \pi i^{t} a w(z)} D_{\infty} \theta\left[\begin{array}{l}
a \\
b
\end{array}\right](w(z))
$$

Since $f\left(z_{1}, z_{0}\right)$ vanishes, $Q\left(z_{1}, z_{2}\right)$ is analytic. From (3) and (4) we have that the factor of automorphy of $f\left(z_{1}, z_{2}\right)$ under the action of $\Gamma$ on $z_{1}$ is $\hat{\rho}_{w\left(z_{2}\right)} \zeta^{2}$. So (6) shows that

$$
Q\left(\gamma z_{1}, z_{2}\right)=\hat{\rho}_{w\left(z_{2}\right)} \zeta\left(\gamma, z_{1}\right) Q\left(z_{1}, z_{2}\right)
$$

and (7) shows that $Q$ is skew-symmetric. Hence $q=C Q$ for some constant $C$ which we now determine up to sign.

Remarks 1) A particular odd theta characteristic was singled out in the definition of $Q$ because we assumed a particular marking for $X$.

2) Formula (6) is similar to one given in [Gu5], where the derivatives are taken with respect to canonical coordinates.

\section{Theorem}

$$
q\left(z_{1}, z_{2}\right)= \pm \frac{e^{\pi i^{t} a \Omega a+2 \pi i^{t} a b} \operatorname{det}(\sigma) \theta\left[\begin{array}{l}
a \\
b
\end{array}\right]\left(w\left(z_{1}\right)-w\left(z_{2}\right)\right)}{D_{2} \theta\left[\begin{array}{c}
a \\
b
\end{array}\right](0) \Sigma\left(z_{1}\right) \Sigma\left(z_{2}\right)} .
$$

Proof We will use (2) to compute $\pm C$. It follows directly from (1) that changing $\eta^{\prime}$ or $\eta^{\prime \prime}$ by an integer vector at most changes the sign of $\theta\left[\begin{array}{c}\eta^{\prime} \\ \eta^{\prime \prime}\end{array}\right](v)$. Since we will only be computing $\pm C$, we will identify theta characteristics modulo 1 , and this will not affect any of the formulas that follow. For $1 \leq i \leq 5$, we define theta characteristics $\eta_{i}$ by setting

$$
w\left(P_{i}\right)=\Omega \eta_{i}^{\prime}+\eta_{i}^{\prime \prime} \bmod L
$$


and $\eta_{i}=\left[\begin{array}{l}\eta_{i}^{\prime} \\ \eta_{i}^{\prime \prime}\end{array}\right]$. Let $\delta=\left[\begin{array}{l}a \\ b\end{array}\right] \bmod 1$. It is standard [Gr] that the six odd theta characteristics are $\delta, \delta+\eta_{i}, 1 \leq i \leq 5$, and the 10 even theta characteristics are $\delta+\eta_{i}+\eta_{j}, 1 \leq i<j \leq 5$. Also $\sum_{i=1}^{5} \eta_{i}=0 \bmod 1$.

We will use the following generalization of Jacobi's derivative formula. If $\nu_{1}, \nu_{2}$ are distinct odd theta characteristics, then

$$
\operatorname{det}\left(\frac{\partial}{\partial v_{n}} \theta\left[\nu_{m}\right](0)\right)_{1 \leq m, n \leq 2}= \pm \pi^{2} \prod_{n=1}^{4} \theta\left[\rho_{n}\right](0),
$$

for some set $\left\{\rho_{n}\right\}$ of even theta characteristics. This is due to Rosenhain, and was generalized to all hyperelliptic Riemann surfaces by Thomae. For a modern reference and further generalizations, see [I].

It can be shown (see [C]) that if $\nu_{1}=\delta, \nu_{2}=\delta+\eta_{i}$, then

$$
\left\{\rho_{n}\right\}=\left\{\delta+\eta_{i}+\eta_{j}, \delta+\eta_{i}+\eta_{k}, \delta+\eta_{i}+\eta_{\ell}, \delta+\eta_{i}+\eta_{m}\right\},
$$

where $\{i, j, k, \ell, m\}=\{1,2,3,4,5\}$. If $\nu_{1}=\delta+\eta_{i}, \nu_{2}=\delta+\eta_{j}$, then

$$
\left\{\rho_{n}\right\}=\left\{\delta+\eta_{i}+\eta_{j}, \delta+\eta_{k}+\eta_{\ell}, \delta+\eta_{k}+\eta_{m}, \delta+\eta_{\ell}+\eta_{m}\right\}
$$

Now plugging $q=C Q$ into (2), we get for any $z, z_{1}, z_{2} \in \tilde{X}$ that

$$
\begin{aligned}
& \theta\left(\Omega a+b+w(z)-w\left(z_{1}\right)-w\left(z_{2}\right)\right) \theta\left[\begin{array}{l}
a \\
b
\end{array}\right]\left(w\left(z_{1}\right)-w\left(z_{2}\right)\right) \Sigma(z)^{2} \\
& =C \operatorname{det}\left(w_{i}^{\prime}\left(z_{j}\right)\right)_{1 \leq i, j \leq 2} \theta\left[\begin{array}{c}
a \\
b
\end{array}\right]\left(w(z)-w\left(z_{1}\right)\right) \theta\left[\begin{array}{l}
a \\
b
\end{array}\right]\left(w(z)-w\left(z_{2}\right)\right) .
\end{aligned}
$$

Now

$$
\begin{aligned}
& w_{i}^{\prime}\left(z_{j}\right)=\lim _{z_{j}^{\prime} \rightarrow z_{j}} \frac{w_{i}\left(z_{j}\right)-w_{i}\left(z_{j}^{\prime}\right)}{q\left(z_{j}, z_{j}^{\prime}\right)}=\frac{\Sigma\left(z_{j}\right)^{2}}{C} \lim _{z_{j}^{\prime} \rightarrow z_{j}} \frac{w_{i}\left(z_{j}\right)-w_{i}\left(z_{j}^{\prime}\right)}{\theta\left[\begin{array}{l}
a \\
b
\end{array}\right]\left(w\left(z_{j}\right)-w\left(z_{j}^{\prime}\right)\right)} \\
& =\frac{\Sigma\left(z_{j}\right)^{2}}{C} \lim _{z_{j}^{\prime} \rightarrow z_{j}} \frac{1}{\frac{\partial}{\partial v_{1}} \theta\left[\begin{array}{l}
a \\
b
\end{array}\right](0) \frac{w_{1}\left(z_{j}\right)-w_{1}\left(z_{j}^{\prime}\right)}{w_{i}\left(z_{j}\right)-w_{i}\left(z_{j}^{\prime}\right)}+\frac{\partial}{\partial v_{2}} \theta\left[\begin{array}{l}
a \\
b
\end{array}\right](0) \frac{w_{2}\left(z_{j}\right)-w_{2}\left(z_{j}^{\prime}\right)}{w_{i}\left(z_{j}\right)-w_{i}\left(z_{j}^{\prime}\right)}} .
\end{aligned}
$$

Using

$$
\lim _{z_{j}^{\prime} \rightarrow z_{j}} \frac{\int_{z_{j}^{\prime}}^{z_{j}} \frac{x d x}{y}}{\int_{z_{j}^{\prime}}^{z_{j}} \frac{d x}{y}}=x\left(z_{j}\right)
$$

we get

$$
\lim _{z_{j}^{\prime} \rightarrow z_{j}} \frac{w_{1}\left(z_{j}\right)-w_{1}\left(z_{j}^{\prime}\right)}{w_{2}\left(z_{j}\right)-w_{2}\left(z_{j}^{\prime}\right)}=\frac{\sigma_{11}+\sigma_{12} x\left(z_{j}\right)}{\sigma_{21}+\sigma_{22} x\left(z_{j}\right)} .
$$


So

$$
\begin{aligned}
& \operatorname{det}\left(\lim _{z_{j}^{\prime} \rightarrow z_{j}} \frac{w_{i}\left(z_{j}\right)-w_{i}\left(z_{j}^{\prime}\right)}{\theta\left[\begin{array}{l}
a \\
b
\end{array}\right]\left(w\left(z_{j}\right)-w\left(z_{j}^{\prime}\right)\right)}\right)_{1 \leq i, j \leq 2} \\
& =\frac{\operatorname{det}\left(\begin{array}{cc}
\sigma_{11}+\sigma_{12} x\left(z_{1}\right) & \sigma_{11}+\sigma_{12} x\left(z_{2}\right) \\
\sigma_{21}+\sigma_{22} x\left(z_{1}\right) & \sigma_{21}+\sigma_{22} x\left(z_{2}\right)
\end{array}\right)}{\prod_{n=1}^{2}\left(\frac{\partial}{\partial v_{1}} \theta\left[\begin{array}{l}
a \\
b
\end{array}\right](0)\left(\sigma_{11}+\sigma_{12} x\left(z_{n}\right)\right)+\frac{\partial}{\partial v_{2}} \theta\left[\begin{array}{l}
a \\
b
\end{array}\right](0)\left(\sigma_{21}+\sigma_{22} x\left(z_{n}\right)\right)\right)} \\
& =\frac{\operatorname{det}(\sigma)\left(x\left(z_{2}\right)-x\left(z_{1}\right)\right)}{\prod_{n=1}^{2}\left(-D_{2} \theta\left[\begin{array}{l}
a \\
b
\end{array}\right](0)-x\left(z_{n}\right) D_{1} \theta\left[\begin{array}{l}
a \\
b
\end{array}\right](0)\right)} \\
& =\operatorname{det}(\sigma)\left(x\left(z_{2}\right)-x\left(z_{1}\right)\right) /\left(D_{2} \theta\left[\begin{array}{l}
a \\
b
\end{array}\right](0)\right)^{2} .
\end{aligned}
$$

Hence putting together (10), (11) and (12), we have

(13)

$$
\begin{aligned}
& C \theta\left(\Omega a+b+w(z)-w\left(z_{1}\right)-w\left(z_{2}\right)\right) \theta\left[\begin{array}{l}
a \\
b
\end{array}\right]\left(w\left(z_{1}\right)-w\left(z_{2}\right)\right) \Sigma(z)^{2}\left(D_{2} \theta\left[\begin{array}{l}
a \\
b
\end{array}\right](0)\right)^{2} \\
& \quad=(\operatorname{det}(\sigma))\left(x\left(z_{2}\right)-x\left(z_{1}\right)\right) \Sigma\left(z_{1}\right)^{2} \Sigma\left(z_{2}\right)^{2} \theta\left[\begin{array}{c}
a \\
b
\end{array}\right]\left(w(z)-w\left(z_{1}\right)\right) \theta\left[\begin{array}{l}
a \\
b
\end{array}\right]\left(w(z)-w\left(z_{2}\right)\right) .
\end{aligned}
$$

Since from (1)

$$
\begin{aligned}
e^{2 \pi i^{t} a\left(w(z)-w\left(z_{1}\right)-w\left(z_{2}\right)\right)} \theta( & \left(\Omega a+b+w(z)-w\left(z_{1}\right)-w\left(z_{2}\right)\right) \\
= & e^{-\pi i^{t} a \Omega a-2 \pi i^{t} a b} \theta\left[\begin{array}{l}
a \\
b
\end{array}\right]\left(w(z)-w\left(z_{1}\right)-w\left(z_{2}\right)\right),
\end{aligned}
$$

using (8) repeatedly we get from (13) that

$$
\begin{gathered}
C^{\prime} \frac{\theta\left[\begin{array}{c}
a \\
b
\end{array}\right]\left(w(z)-w\left(z_{1}\right)-w\left(z_{2}\right)\right) D_{\infty} \theta\left[\begin{array}{c}
a \\
b
\end{array}\right](w(z))}{\theta\left[\begin{array}{c}
a \\
b
\end{array}\right]\left(w(z)-w\left(z_{1}\right)\right) \theta\left[\begin{array}{l}
a \\
b
\end{array}\right]\left(w(z)-w\left(z_{2}\right)\right)} \frac{\theta\left[\begin{array}{c}
a \\
b
\end{array}\right]\left(w\left(z_{1}\right)-w\left(z_{2}\right)\right)}{D_{\infty} \theta\left[\begin{array}{c}
a \\
b
\end{array}\right]\left(w\left(z_{1}\right)\right) D_{\infty} \theta\left[\begin{array}{c}
a \\
b
\end{array}\right]\left(w\left(z_{2}\right)\right)} \\
=(\operatorname{det}(\sigma))\left(x\left(z_{2}\right)-x\left(z_{1}\right)\right) /\left(D_{2} \theta\left[\begin{array}{l}
a \\
b
\end{array}\right](0)\right)^{2},
\end{gathered}
$$

where $C^{\prime}=C e^{-\pi i^{t} a \Omega a-2 \pi i^{t} a b}$.

At this point we square (14), and let $z, z_{1}, z_{2}$ be any points such that $\Pi(z)=$ $P_{k}, \Pi\left(z_{1}\right)=P_{i}, \Pi\left(z_{2}\right)=P_{j}$, for distinct $i, j, k \in\{1,2,3,4,5\}$. Then using (1) repeatedly, from (14) we have

$\left(C^{\prime}\right)^{2} \frac{\theta\left[\delta+\eta_{\ell}+\eta_{m}\right](0)^{2} D_{\infty} \theta\left[\delta+\eta_{k}\right](0)^{2}}{\theta\left[\delta+\eta_{i}+\eta_{k}\right](0)^{2} \theta\left[\delta+\eta_{j}+\eta_{k}\right](0)^{2}} \frac{\theta\left[\delta+\eta_{i}+\eta_{j}\right](0)^{2}}{D_{\infty} \theta\left[\delta+\eta_{i}\right](0)^{2} D_{\infty} \theta\left[\delta+\eta_{j}\right](0)^{2}}$

$$
=(\operatorname{det}(\sigma))^{2}\left(a_{i}-a_{j}\right)^{2} /\left(D_{2} \theta[\delta](0)\right)^{4},
$$


where $\{i, j, k, l, m\}=\{1,2,3,4,5\}$. We will now apply Rosenhain's formula (9). Since $D_{\infty} \theta[\delta](0)=0$, we have

$$
\begin{aligned}
& D_{2} \theta[\delta](0)^{2} D_{\infty} \theta\left[\delta+\eta_{k}\right](0)^{2}=\operatorname{det}\left(\begin{array}{cc}
D_{2} \theta[\delta](0) & D_{2} \theta\left[\delta+\eta_{k}\right](0) \\
D_{1} \theta[\delta](0) & D_{1} \theta\left[\delta+\eta_{k}\right](0)
\end{array}\right)^{2} \\
& =(\operatorname{det}(\sigma))^{2} \operatorname{det}\left(\begin{array}{cc}
\frac{\partial}{\partial \nu_{1}} \theta[\delta](0) & \frac{\partial}{\partial v_{1}} \theta\left[\delta+\eta_{k}\right](0) \\
\frac{\partial}{\partial v_{2}} \theta[\delta](0) & \frac{\partial}{\partial v_{2}} \theta\left[\delta+\eta_{k}\right](0)
\end{array}\right)^{2} \\
& =(\operatorname{det}(\sigma))^{2} \pi^{4} \theta\left[\delta+\eta_{k}+\eta_{i}\right](0)^{2} \theta\left[\delta+\eta_{k}+\eta_{j}\right](0)^{2} \\
& \theta\left[\delta+\eta_{k}+\eta_{\ell}\right](0)^{2} \theta\left[\delta+\eta_{k}+\eta_{m}\right](0)^{2} .
\end{aligned}
$$

Similarly, (5) and Riemann's vanishing theorem imply that $D_{a_{i}} \theta\left[\delta+\eta_{i}\right](0)=0$, so $D_{a_{j}} \theta\left[\delta+\eta_{i}\right](0)=\left(a_{j}-a_{i}\right) D_{\infty} \theta\left[\delta+\eta_{i}\right](0)$. Hence, reasoning as in (16), by (9),

$$
\begin{aligned}
D_{\infty} \theta\left[\delta+\eta_{i}\right](0)^{2} D_{\infty} \theta\left[\delta+\eta_{j}\right](0)^{2} & \left(a_{i}-a_{j}\right)^{-4} D_{a_{j}} \theta\left[\delta+\eta_{i}\right](0)^{2} D_{a_{i}} \theta\left[\delta+\eta_{j}\right](0)^{2} \\
= & \left(a_{i}-a_{j}\right)^{-2} \operatorname{det}\left(\begin{array}{cc}
D_{2} \theta\left[\delta+\eta_{i}\right](0) & D_{2} \theta\left[\delta+\eta_{j}\right](0) \\
D_{1} \theta\left[\delta+\eta_{i}\right](0) & D_{1} \theta\left[\delta+\eta_{j}\right](0)
\end{array}\right)^{2} \\
= & \left(a_{i}-a_{j}\right)^{-2}(\operatorname{det}(\sigma))^{2} \operatorname{det}\left(\begin{array}{ll}
\frac{\partial}{\partial v_{1}} \theta\left[\delta+\eta_{i}\right](0) & \frac{\partial}{\partial v_{1}} \theta\left[\delta+\eta_{j}\right](0) \\
\frac{\partial}{\partial v_{2}} \theta\left[\delta+\eta_{i}\right](0) & \frac{\partial}{\partial v_{2}} \theta\left[\delta+\eta_{j}\right](0)
\end{array}\right)^{2} \\
= & \left(a_{i}-a_{j}\right)^{-2}(\operatorname{det}(\sigma))^{2} \pi^{4} \theta\left[\delta+\eta_{i}+\eta_{j}\right](0)^{2} \\
& \quad \theta\left[\delta+\eta_{k}+\eta_{\ell}\right](0)^{2} \theta\left[\delta+\eta_{k}+\eta_{m}\right](0)^{2} \theta\left[\delta+\eta_{\ell}+\eta_{m}\right](0)^{2} .
\end{aligned}
$$

Combining (15), (16), and (17) we get

$$
\left(C^{\prime}\right)^{2}=(\operatorname{det}(\sigma))^{2} /\left(D_{2} \theta[\delta](0)\right)^{2},
$$

so $C= \pm e^{\pi i^{t} a \Omega a+2 \pi i^{t} a b} \operatorname{det}(\sigma) / D_{2} \theta[\delta](0)$, which gives us our theorem.

Remarks 1) Although affine transformations $(x, y) \rightarrow\left(\alpha^{2} x+\beta, \alpha^{5} y\right)$ of our curve affect the differential operators $D_{1}, D_{2}$, they leave $\operatorname{det}(\sigma) / D_{2} \theta[\delta](0) \Sigma\left(z_{1}\right) \Sigma\left(z_{2}\right)$ invariant.

2) The constant $D_{2} \theta[\delta](0)$ is related to the discriminant of our curve: see [Gr].

Acknowledgements I would like to thank Cris Poor for helpful conversations on this material and his comments on an earlier version of this paper. I would also like to thank Columbia University, whose hospitality I was enjoying while working on part of this paper. 


\section{References}

[C] G. Coogan, A generalization of Jacobi's derivative formula. Ph.D. Thesis, University of Colorado at Boulder, 1999.

[F] J. Fay, Theta Functions on Riemann surfaces. Lecture Notes in Math. 353, Springer-Verlag, Berlin, 1973.

[Gr] D. Grant, A generalization of Jacobi's derivative formula to dimension two. J. Reine Angew. Math. 392(1988), 125-136.

[Gu1] R. C. Gunning, Riemann Surfaces and Generalized Theta Functions. Springer-Verlag, Berlin, 1976.

[Gu2] — On generalized theta functions. Amer. J. Math. (1) 104(1982), 183-208.

[Gu3] - Some identities for abelian integrals. Amer. J. Math. 108(1986), 39-74.

[Gu4] - Analytic identities for theta functions. Proc. Symp. Pure Math. (1) 49(1989), 503-515.

[Gu5] W Notes from a course given at Princeton University. 1987-88.

[I] J-I. Igusa, On Jacobi’s Derivative Formula and its generalizations. Amer. J. Math. (2) 102(1980), 409-446.

[M] D. Mumford, Tata Lectures on Theta, I, II. Prog. in. Math. 28, 43, Birkhauser, Boston, 1983, 1984.

[P] C. Poor, The hyperelliptic locus. Duke Math. J. (3) 76(1994), 809-884.

Department of Mathematics

University of Colorado at Boulder

Boulder, Colorado 80309-0395

U.S.A.

e-mail: grant@boulder.colorado.edu 